\title{
Designing Mobility Models Based on Relational Graph
}

\author{
Zhenwei DING $^{\dagger \text { a) }}$, Yusuke OMORI ${ }^{\dagger b)}$, Student Members, Ryoichi SHINKUMA ${ }^{\dagger c)}$, Senior Member, \\ and Tatsuro TAKAHASHI ${ }^{\dagger \mathrm{d})}$, Fellow
}

\begin{abstract}
SUMMARY Simulating the mobility of mobile devices has always been an important issue as far as wireless networks are concerned because mobility needs to be taken into account in various situations in wireless networks. Researchers have been trying, for many years, to improve the accuracy and flexibility of mobility models. Although recent progress of designing mobility models based on social graph have enhanced the performance of mobility models and made them more convenient to use, we believe the accuracy and flexibility of mobility models could be further improved by taking a more integrated structure as the input. In this paper, we propose a new way of designing mobility models on the basis of relational graph [1] which is a graph depicting the relation among objects, e.g. relation between people and people, and also people and places. Moreover, some novel mobility features were introduced in the proposed model to provide social, spatial and temporal properties in order to produce results similar to real mobility data. It was demonstrated by simulation that these measures could generate results similar to real mobility data.

key words: mobility model, relational graph, social graph, contact duration, inter-contact time
\end{abstract}

\section{Introduction}

In recent years, ad-hoc communications such as Mobile Ad-hoc Networks (MANET), Vehicular Ad-hoc Networks (VANET) [2] and Opportunistic Networks [3] have drawn the attention of many researchers, and a variety of routing protocols and applications were put forward for these networks in the literature. However, in view of the intrinsic characteristic of mobility within these networks, the feasibility of such protocols and applications must be proved in the context of realistic movement of wireless devices.

Although the transmission of data is conducted by wireless devices, in the research of mobility of wireless devices, attention is focused on people because mobile devices are carried by people most of the time, so the mobility problem of wireless devices is actually the mobility problem of people.

Although a number of experiments have been carried out to collect real movement traces in the past, real mobility datasets have rarely been used for the evaluation and testing of protocols and applications for wireless networks [4] due to the difficulty in implementation and lack of generality. In

Manuscript received January 7, 2014.

Manuscript revised May 24, 2014.

${ }^{\dagger}$ The authors are with the Graduate School of Informatics, Kyoto University, Kyoto-shi, 606-8501 Japan.

a)E-mail: ding@cube.kuee.kyoto-u.ac.jp

b)E-mail: omori@cube.kuee.kyoto-u.ac.jp

c)E-mail: shinkuma@i.kyoto-u.ac.jp

d) E-mail: ttakahashi@i.kyoto-u.ac.jp

DOI: $10.1587 /$ transinf.2014PAP0004 contrast, synthetic mobility models are generally preferred due to their theoretical attributes, which make them relatively easier to use.

The earliest and most widely used mobility model is the random waypoint model [5] in which a person's speed, direction, and destination are assumed to be random. It is widely used in simulation and evaluation because of its simplicity. However, it fails to consider any practical influence on a person's movement, and the results of this model were proved to be largely at variance with real mobility data [6].

Researchers have since identified temporal and spatial properties such as location preferences and difference between weekday and weekend that affect a person's movements. Their incorporation has made mobility models more accurate and reality-oriented [7].

Recently, researchers have found that a person's social relation has great influence on his or her movements, prompting the emergence of social mobility models which concentrate primarily on people's social networks. In previous studies of social mobility models, social relationships were assumed to be the driving force of a person's movements. However, social relationships cannot account for all aspects of human movement. For example, the notion of familiar strangers [8] states that some people frequently cross paths although they do not actually know each other. It is estimated that social relationships can explain only about $10 \%$ of a person's movements as derived from cell phone data and up to $30 \%$ as derived from location-based social networks [9]. Therefore, social, spatial as well as temporal properties all play important roles in the building of mobility models. However, most mobility models consider only one or two properties, and for those which do consider all properties, they don't have an integrated structure to deal with different properties. Such an integrated structure is beneficial to consider time variances of spatial and social factors, which have some correlation; for example, the social and spatial factors of a person on weekdays would be affected by her or his work style, while those factors on weekend would be affected by her or his private life.

In this paper, we propose a novel way of modeling human movement on the basis of relational graph. Relational graph is a graph depicting the relation among objects (e.g. relation between people and people, and also people and places). We believe that relational graph could facilitate the usage of mobility models compared with social graph by incorporating social properties and other properties includ- 
ing spatial and temporal properties altogether. Since as we mentioned above, any prior work did not present such an integrated structure of mobility, to the best of our knowledge, this is the world-first one.

Furthermore, we propose some new spatial and temporal properties based on real life expefrience and some new metrics for evaluating mobility models which we think could have significant value in the simulation and evaluation of routing protocols in MANET or VANET. Simulation demonstrated that these measures have produced results similar to real mobility data.

The structure of this paper is as follows: Section 2 gives a brief overview of recent related work in the research of mobility models. Section 3 gives the definition and details of relational graph used in this research. Section 4 describes the system model of our proposed mobility model. Section 5 compares the simulation results of our proposed model with real mobility data and previous mobility model in both conventional metrics and new metrics proposed in this paper. Section 6 concludes with a summary of the key points and a discussion of possible future research directions.

\section{Related Work}

In this section, we discuss recent progress in the research of mobility models.

The first mobility model putting forward the idea that mobility models should incorporate social relations is the Community-based Mobility Model (CMM) [4]. CMM is based on the assumption that the mobility patterns of devices are driven by people's movement and the main factor controlling a person's movements is his or her social relationships. This model generates social network using social network theory and detects communities by community detection algorithm. Then each community is associated to a certain location, and the nodes representing people move toward their goal which is calculated from the ratio of social relationships.

Several improved models of CMM have thus been put forward. The Home-cell Community-based Mobility Model [10] (HCMM) introduced the properties of location attraction and preference for short distances into the community-based mobility model. In HCMM, every user is assumed to belong to one main social community and a home cell is assigned to each social community. The home cell has strong attraction toward those nodes which are assigned to it while other cells only have a relatively low attraction toward those nodes. HCMM is the first mobility model which combines social attraction together with spatial attraction.

The Enhanced Community-based Mobility Model [11] (ECMM) further enhances both the accuracy and flexibility of community-based mobility models. By introducing mobility characteristics like pause time and group movement, ECMM has achieved a result more similar to real mobility dataset than previous community-based mobility models. By separating the social network model from the con- ventional community-based mobility model, ECMM is able to take arbitrary social networks as its input, thus enabling it to generate mobility traces of arbitrary social networks.

Besides the community-based mobility models, there are other mobility models which center on social relations. Daniel Fischer et al. presented a General Social Mobility Model (GeSoMo)[12] which is the first mobility model leveraging the concept of separating simulation model from the input data of social network, thus improving the flexibility of GeSoMo. In GeSoMo, location attraction and social attraction were also introduced, moreover, a new feature of repulsion between nodes was introduced to simulate the phenomenon of avoiding meeting people with weak social relation. Ana Gainaru et al. [13] designed a mobility model using social networks for the simulation of VANETs which takes into account the social relationship between vehicles. Their mobility model automatically generates entry and exit points for all vehicles and then designs the routes for all of these points. Vincent Borrel et al. proposed SIMPS [6] which was based on human feature in sociology that each individual will try to regulate her socialization to her own sociability by the effect of her actions. SIMPS consists of two parts, social motion influence and motion execution unit which exerts social and spatial influence on the movement of human beings.

Apart from social mobility models which regard social relation as the main driving force of people's movement, some mobility models focus on spatial properties while others focus on temporal properties.

The working day movement model [14] put forward by Frans Ekman et al. captured several different mobility features at a lower level by combining different movement model referred as submodel. Wei-Jen Hsu et al. proposed Time-Variant Community mobility model (TVC) [15] which focuses on periodical re-appearance at same locations and skewed location visiting preferences observed in mobility dataset.

P. Venkateswaran et al. [16] proposed a mobility model in MANET which focused mainly on spatial properties like pause time and obstacles. Kyunghan Lee et al. presented SLAW [17] which captures spatial mobility features like power-law flights and fractal waypoints in mobility traces. Power-law flights mean the power-law distribution of human walks in pause time. Fractal waypoints mean people are always more likely to be attracted to more popular places. Alessandro Mei et al. proposed SWIM [18] which is a simple mobility model for ad hoc network. SWIM was designed using simple and clear algorithms which lack in social and temporal properties of human movement.

\section{Relational Graph}

\subsection{Definition of Relational Graph}

In this subsection, we define the concept of relational graph. Relational graph is a graph which describes the relation between not only people and people, but also between people 


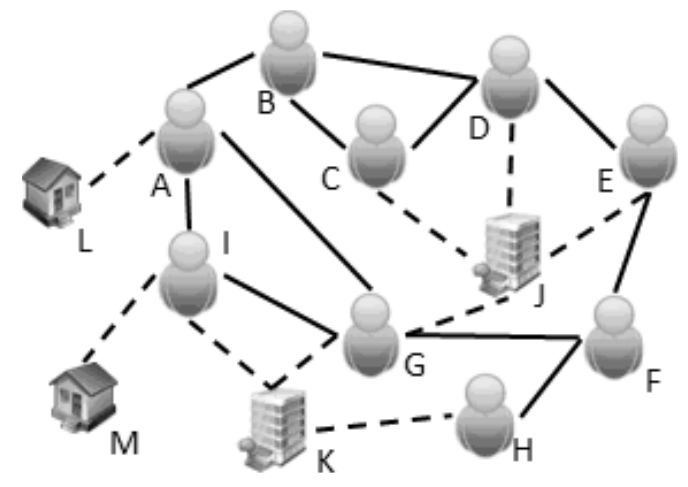

Fig. 1 An example of relational graph.

and other objects, for example people and places. In previous researches of mobility models, social graph was taken as the input for mobility models. Relational graph can be considered as a further development from social graph which only depicts the relation between people and people. And we believe that relational graph could facilitate the usage of mobility models compared with social graph by incorporating social properties and other properties including spatial and temporal properties altogether.

Figure 1 shows an example of relational graph. In the relational graph, nodes can represent people or places compared with that of social graph where nodes only represent people. In Fig. 1, solid lines describe the relation between people and people, and dashed lines describe the relation between people and places. In social graph, a number ranging between 0 and 1 is given to each edge, and is referred as weight. The weights associated with each edge of the network are used to model the strength of the interactions between individuals [19]. Same as the social graph, our relational graph also uses a number ranging between 0 and 1 to model the strength of interactions. However, since nodes can represent people or places in relational graph, the strength of interactions not only includes interactions between people and people but also includes interactions between people and places.

While social graph can be represented by a matrix when used as input of mobility models, relational graph requires two or more matrices in order to represent both the relation between people and people, and relation between people and other objects.

Figure 2 shows an example of using matrices to represent the relational graph in Fig. 1. Matrix $M_{1}$ describes the relation between people and people. Matrix $M_{2}$ describes the relation between people and places. In Matrix $M_{1}$ both rows and columns represent people while in Matrix $M_{2}$ columns represent people and rows represent places.

\subsection{Generating Relational Graph Using Lifemap Dataset}

In our mobility model, the input data of relational graph was calculated from a mobility dataset called Lifemap [20]. The reason why we chose Lifemap is that this mobility dataset

$$
\begin{aligned}
M_{1} & =\left[\begin{array}{ccccccccc}
1 & 0.13 & 0 & 0 & 0 & 0 & 0.1 & 0 & 0.32 \\
0.13 & 1 & 0.34 & 0.5 & 0 & 0 & 0 & 0 & 0 \\
0 & 0.34 & 1 & 0.41 & 0 & 0 & 0 & 0 & 0 \\
0 & 0.5 & 0.41 & 1 & 0.05 & 0 & 0 & 0 & 0 \\
0 & 0 & 0 & 0.05 & 1 & 0.71 & 0 & 0 & 0 \\
0 & 0 & 0 & 0 & 0.71 & 1 & 0.07 & 0.19 & 0 \\
0.1 & 0 & 0 & 0 & 0 & 0.07 & 1 & 0 & 0.55 \\
0 & 0 & 0 & 0 & 0 & 0.19 & 0 & 1 & 0 \\
0.32 & 0 & 0 & 0 & 0 & 0 & 0.55 & 0 & 1
\end{array}\right] \\
M_{2} & =\left[\begin{array}{ccccccccc}
0 & 0 & 0.25 & 0.34 & 0.84 & 0 & 0.79 & 0 & 0 \\
0 & 0 & 0 & 0 & 0 & 0 & 0.13 & 0.68 & 0.51 \\
0.63 & 0 & 0 & 0 & 0 & 0 & 0 & 0 & 0 \\
0 & 0 & 0 & 0 & 0 & 0 & 0 & 0 & 0.12
\end{array}\right]
\end{aligned}
$$

Fig. 2 Matrix representation of relational graph.

not only consists of location information like identities of AP but it also contains specific geographical information which is the longitude and latitude of each place, making it easier in terms of simulation.

A record of the Lifemap dataset consists of user ID, ID of the access point the user is associated with, and the start and end time of the association. User IDs and access point IDs become nodes of people and places in the relational graph, respectively. The method of getting the relational graph is that first we took the first one quarter of mobility data of every person in Lifemap based on temporal sequence. Then we calculated and enumerated all contacts between people and people as well as contacts between people and places, and how long the duration of those contacts are. Finally, we have the duration of each contact enumerated in step two divided by a threshold $\lambda$ (e.g. 1800 seconds) to get an averaged value and sum up the value of the items representing contacts between same person and person or same person and place, and let each averaged value divided by the sum of all averaged value of a certain person that value is involved to get a weight of an edge in the relational graph.

The weight of the edge between nodes $i$ and $j, w_{i, j}$ is defined as:

$$
w_{i, j}=\frac{\left[\frac{\mathrm{CD}_{i, j}}{\lambda}\right]}{\sum_{k \in M_{1}}\left[\frac{\mathrm{CD}_{i, k}}{\lambda}\right]+\sum_{k \in M_{2}}\left[\frac{\mathrm{CD}_{i, k}}{\lambda}\right]}
$$

where $\mathrm{CD}_{i, j}$ is a total time of contact durations between nodes $i$ and $j$. Lambda is a parameter. $[X]$ means the Gaussian symbol; it indicates the maximum integer that is equal or smaller than $X$. If nodes $i$ and $j$ are people, $w_{i, j}$ goes to $M_{1}$, while it goes to $M_{2}$ if node $j$ is a place.

\section{System Model}

In order to make our model easier to use, our model inherited the feature of separating the input data from the mobility model put forward by Fischer et al. [12]. After having 


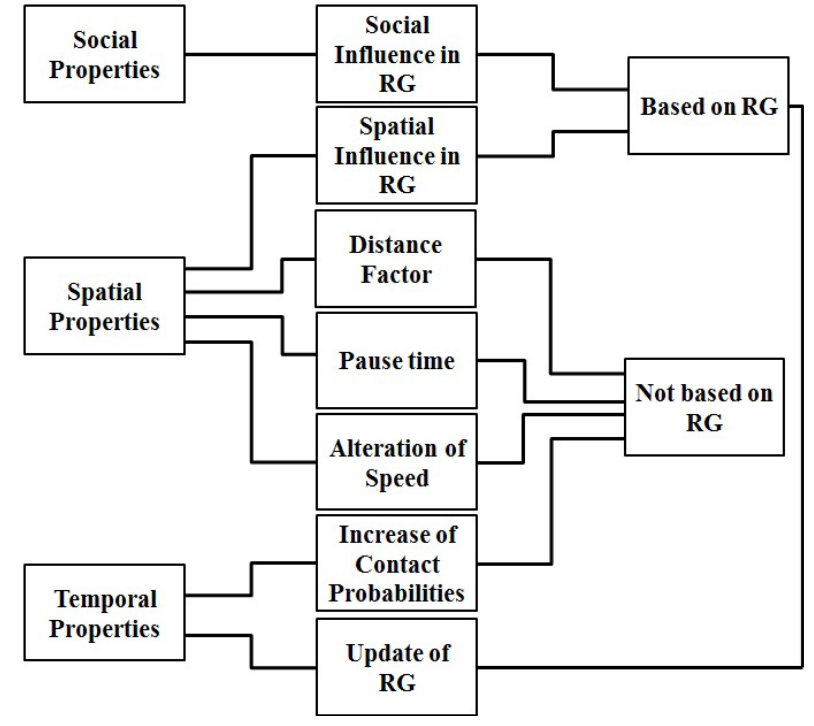

Fig.3 Mobility features comprised and their links with relational graph.

the input information of relational graph, a number of nodes representing people are generated and their related information is initialized. At first, each node is placed randomly then a goal is given to each node, and during each time slot, each node moves toward its goal. After reaching its goal, a node stays there for a period of time. It leaves for the next goal in accordance with a goal deciding algorithm, which will be discussed in detail in the following paragraphs.

In our model, people and places are represented by nodes just as how they are represented in the relational graph. However, only those nodes representing people are able to move in the mobility model. The entire map is made up of many grids which could be understood as a block in the real map. The probability of a node $i$ deciding node $k$ as the destination is referred as $P($ goal $=$ node $k$ ), and this probability could be influenced by the relation between these two nodes in the relational graph referred as $P_{r}($ goal $=$ node $k)$ and the distance between these two nodes in the mobility model referred as $P_{d}$ (goal $=$ node $k$ ). The detailed equation will be explained in the following subsections.

Figure 3 shows the mobility features in our model and their links with relational graph. In Fig. 3, "RG" means Relational Graph. The details of these mobility features are explained in the following subsections.

\subsection{Social Properties}

Since relational graph consists of social information, therefore our model is influenced by the social relation between people and people. However, since our relational graph combines together social information like relation between people and people and spatial information like relation between people and places, the algorithm of deciding the destination according to relational graph involves both relation between people and people and relation between people and places. The influence of relational graph on the probability of a node $i$ deciding node $k$ as the destination is as follows:

$$
P_{r}(\text { goal }=\text { node } k)=\frac{w_{1_{i, k} / 2_{i, k}}}{\sum_{j \in M_{1}} w_{i, j}+\sum_{j \in M_{2}} w_{i, j}}
$$

where $w_{i, j}$ means the weight between node $i$ and node $j$ in relational graph, and $M_{1}$ represents the matrix describing relation between people and people while $M_{2}$ represents the matrix describing relation between people and places.

In Eq. (2), ' $/$ ' means 'or'. That is, if nodes $i$ and $j$ are people, $w_{i, j}$ goes to $M_{1}$, while $w_{i, j}$ goes to $M_{2}$ if node $j$ is a place.

The probability of a node $i$ deciding node $k$ as the destination in terms of relational influence is the proportion of the weight between node $i$ and node $k$ either in $M_{1}$ or $M_{2}$, because node $k$ could be either people or place, to the sum of weight between node $i$ and all other nodes both in $M_{1}$ and $M_{2}$. If node $k$ is a person, then the destination of node $i$ will be the current destination of node $k$. If node $k$ is a place, then the destination of node $i$ will be just the place that node $k$ is representing.

\subsection{Spatial Properties}

\subsubsection{Spatial Influence in Relational Graph}

As mentioned in the previous section, since relational graph contains relation between people and places, the influence of people's spatial preference can be represented by relational graph together with social relation. As shown in Eq. (1), spatial influence is described by matrix $M_{2}$ which depicts relation between people and places.

\subsubsection{Distance Factor}

In real life, people generally prefer to go to closer places than to more distant places [18]. The factor of distance attraction is introduced to simulate this preference.

We found that the mechanism of distance attraction follows a power law distribution with a negative exponent. The equation is calculated from statistical analysis of data of the travel distance in NHTS [21]. Then the influence of distance on the probability of a node $i$ deciding node $k$ as the destination is as follows:

$$
P_{d}(\text { goal }=\text { node } k)=\frac{a x_{i, k}^{-b}}{\sum_{j \in M_{1}} a x_{i, j}^{-b}+\sum_{j \in M_{2}} a x_{i, j}^{-b}}
$$

where $x$ refers to the distance between nodes and constant $a$ and $b$ are calculated from the fitted curve of NHTS data produced by MATLAB as shown in Fig. 4. In a practical use of our method, we might need to conduct a survey like NHTS in the region because NHTS might not be applicable for other regions than the United States.

The probability of a node $i$ deciding node $k$ as the destination in terms of distance influence is the proportion of the probability of node $i$ going to node $k$ in the power law 


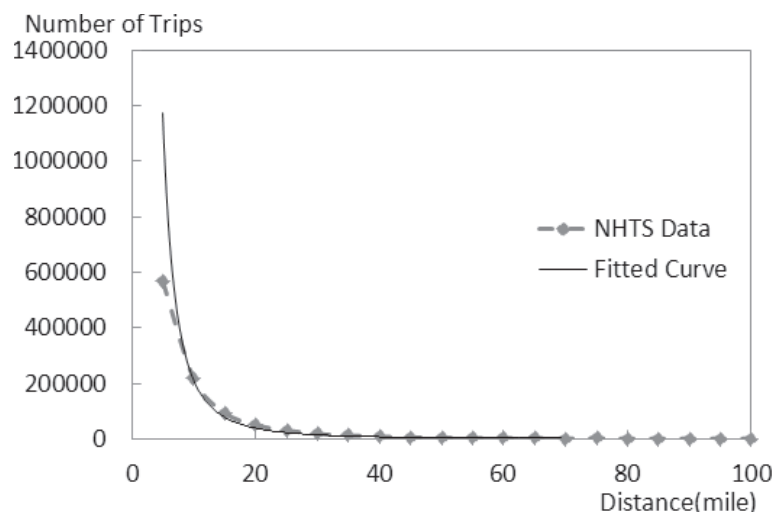

Fig. 4 Power law distribution of distance influence.

equation of distance influence to the sum of the probability of node $i$ going to each node in the relational graph in the power law equation of distance influence.

\subsubsection{Pause Time}

In real life, people usually stay for a period of time before starting to move again when they arrive at a place. This phenomenon is a highly important aspect in real mobility, and it is represented by pause time in this model. After one node reaches its current goal, the node will stay at that place for a period of time instead of starting another movement immediately.

In order to get a more accurate mathematical equation of pause time, we made a statistical analysis of the pause time of the Lifemap dataset. As will be discussed later, since we also use this mobility dataset as the comparison of the simulation results, the analysis of pause time was based only on part of this dataset. By analyzing the dataset, we discovered that basically there are two kinds of pause pattern. One is that most of the time people tend to stay at most places for only a short period of time, and the other one pattern is that people tend to stay at one or two places (e.g. home or work place) for very long time. We label nodes representing the former places as non-key nodes and nodes representing the latter places as key nodes. Then we found that the distribution of pause time for both key nodes and non-key nodes are the superposition of several Gaussian distributions as shown in Fig. 5. Therefore, the pause time in our model is predicated on a mathematical equation which is the superposition of several Gaussian distributions using mathematical software like MATLAB. However, when we practically use our model in a region, we would have to first observe actual mobility in the region to obtain the distribution of pause time.

\subsubsection{Alteration of Speed}

As mentioned by [22], and to the best of our knowledge there is no mobility model which includes the alteration of speed so far. In our model, the alteration of moving speed was introduced, the mechanism of which is nodes have high speed when they are distant from their destination and their

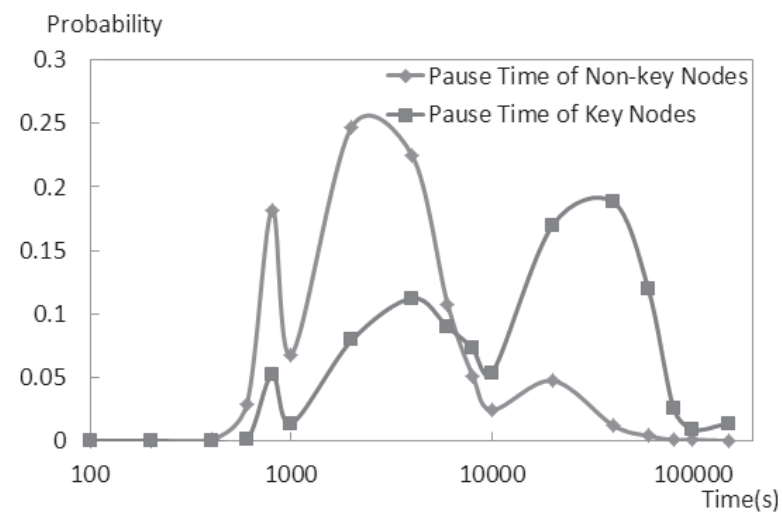

Fig. 5 Pause time of key nodes and non-key nodes.

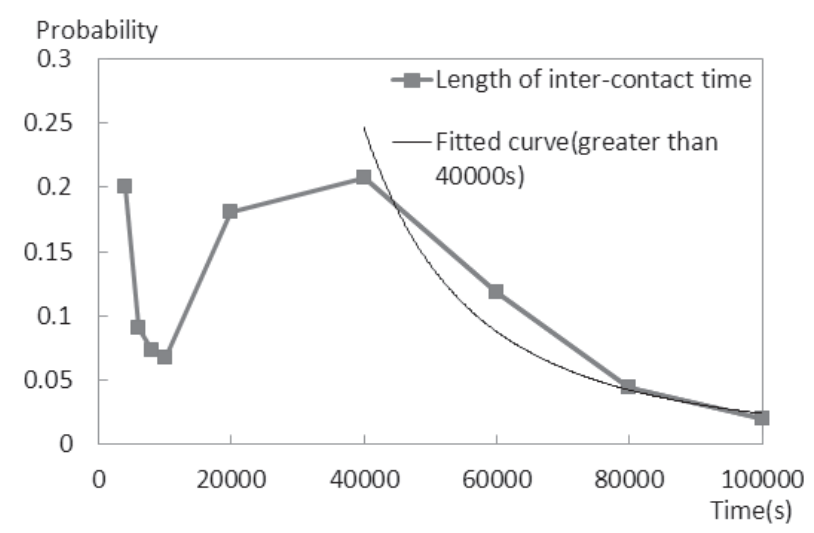

Fig. 6 Probability of inter-contact time between people and their key nodes in each length.

speed will be reduced when they are within 1000 meters from their destination. This is to simulate the phenomenon in real life that people travel by cars or subways or other high speed transportation methods at first and then lower their speed (e.g. walking) before reaching the final goal.

\subsection{Temporal Properties}

\subsubsection{Increase of Contact Probabilities}

Another phenomenon we found by analyzing the Lifemap dataset was that people tend to revisit some places frequently, and these places are exactly the same as the places referred as key nodes in the previous subsection. This phenomenon could be explained as people's regular shuttling of home and work place in real life.

In order to simulate this phenomenon, we took part of the Lifemap dataset and added up the number of intercontact time (time duration between consecutive contact) in each length and calculated the probability. As shown in Fig. 6, after time $=40000$ s which is roughly half a day, the probability of inter-contact time declines as the time length increases which indicates that the probability of people going to home or work places increases as time went on. Therefore, we introduced a power law equation which 
is the $y$-axis symmetry of the fitted curve shown in Fig. 6 to raise the probability of contact between people and their key nodes as time passing by.

\subsubsection{Update of Relational Graph}

As put forward by [23], the input of mobility model might change over time. In order to get the results that are closer to the Lifemap dataset in long term situation, it is of great importance to update the input data since relation between people and people or between people and places could vary with time. The relational graph in our model is updated whenever there is a contact between nodes whose duration are greater than the threshold $\lambda$ mentioned in Sect. 3 .

\subsection{Goal Deciding}

The calculation of the destination of a node is based on both the influence of relational graph and the influence of distance. The probability of a node $i$ deciding node $k$ as the destination is as follows:

$$
\begin{array}{r}
P(\text { goal }=\text { node } k)=\alpha * P_{r}(\text { goal }=\text { node } k)+ \\
\beta * P_{d}(\text { goal }=\text { node } k)
\end{array}
$$

where $\alpha$ and $\beta$ are constant that influence the weight of relational graph and distance. It should be pointed out that since the sum of all probabilities must be less than or equal to 1 so the sum of $\alpha$ and $\beta$ must be 1 . Since we suppose that both relational graph and distance have the same influence, therefore the value of $\alpha$ and $\beta$ is equal in our model.

We will prove this assumption is correct by the simulation results in the following section. However, it should be left as a future work how to optimize $\alpha$ and $\beta$ because it might depend on the region we are going to apply our model to.

\section{Simulation and Evaluation}

The objective of this section is to prove that the proposed model can reproduce characteristics of a real mobility dataset from simulation results. In the simulation of our model, the mobility dataset Lifemap mentioned in the previous section was used for the assessment of the proposed model. Therefore, we set our simulation parameters as relevant as possible to the parameters of Lifemap. The simulation of our mobility model was carried out in a map of $88 \mathrm{~km} \times 110 \mathrm{~km}$, and the size of each grid was $220 \mathrm{~m} \times 220 \mathrm{~m}$. The number of nodes representing people was 67 while the number of nodes representing places was 4512 , and the range of transmission for a mobile device was considered to be 220 meters. The speed of the nodes was randomly distributed from $10 \mathrm{~m} / \mathrm{s}$ to $25 \mathrm{~m} / \mathrm{s}$ when the nodes were far from the destination, and when the nodes were close to the destination, the speed range changed to $1 \mathrm{~m} / \mathrm{s}$ to $5 \mathrm{~m} / \mathrm{s}$. The duration of simulation was 2 months.

Although the input data of relational graph and some of our analysis were based on Lifemap, it should be noted that the relational graph and the analysis were made from the first one quarter of Lifemap based on temporal sequence which is part of the Lifemap dataset not the entire dataset. The reason why the first one quarter of Lifemap is used is that it contains enough mobility data to produce relational graph while the least possible quantity of Lifemap dataset is used since Lifemap dataset is also taken as comparison. And we will compare the results of our mobility model with the entire Lifemap dataset.

In order to measure the performance of our mobility model, we used the metrics of inter-contact time and contact duration. Inter-contact time is the time duration between two consecutive contacts of the same people. Contact duration is the time duration of one contact. Both metrics are of great importance in ad hoc networks, and particularly in opportunistic mobile network [24]. Contact duration represents the length of a contact, therefore affecting the total amount of information that could be transmitted during a contact. Inter-contact time often indicates the frequency and probability of being in contact, thus strongly influencing the speed of relaying information.

Apart from the results of our mobility model and the Lifemap dataset, the result of the generally accepted mobility model CMM was also introduced as a bench mark to show other mobility models can not easily reproduce the characteristics of the Lifemap dataset. Complementary $\mathrm{Cu}-$ mulative Distribution Function (CCDF) was used to show the distribution of probabilities of contact duration and intercontact time.

Figures 7 and 8 show the CCDF of contact duration and inter-contact time between people and people of our proposed model, CMM and different editions of Lifemap in terms of time period. In these figures, " $1 / 4$ of Lifemap" means results from the first one quarter of Lifemap based on temporal sequence. " $1 / 2$ of Lifemap" means results from the first half of Lifemap based on temporal sequence. "3/4 of Lifemap" means results from Lifemap except the last one quarter based on temporal sequence. The reason why the results of different editions of Lifemap are given will be explained later. From these figures, it is noticeable that the

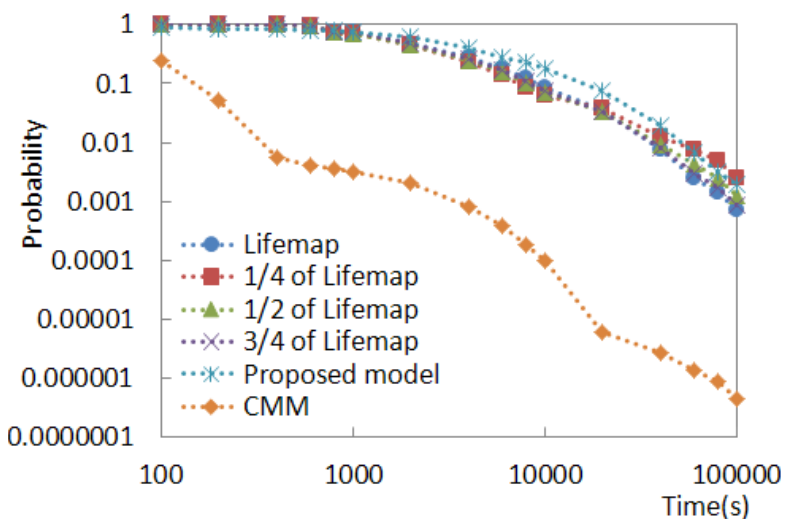

Fig. 7 Contact duration between people and people. 


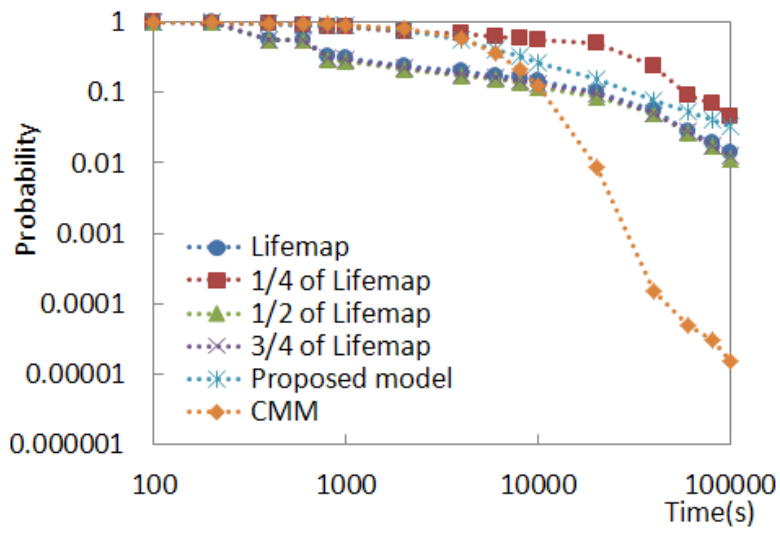

Fig. 8 Inter-contact time between people and people.

results of the proposed model are close to the results of Lifemap dataset where the results of CMM are not the same as the results of the other two. We think that CMM's excessive focus on social property might account for the differences.

However, it is noted that the curve representing one quarter of Lifemap is little different from the other curves representing Lifemap. In Fig. 8, there are less inter-contacts from time $=400$ s to time $=2000$ s than other editions of Lifemap while in Fig. 7, although slightly, there are more contacts after time $=100000$ s than other editions of Lifemap. Therefore, we took a close look at the Lifemap dataset and found that there was a problem caused by AP handover when calculating contact duration and intercontact time between people and people for the Lifemap dataset. When people go from regions to regions which are sensored by different APs, although they are still in contact in real life, it looks like two or more contacts in the dataset. This problem reduces the probability of long time contact duration and increases the probability of short time intercontact time, thus causing some errors when using contact duration and inter-contact time between people and people as metrics. Because the time span of one quarter of Lifemap is shorter than other editions of Lifemap, it contains less long contacts where people moving among different APs, therefore it is less influenced by AP handover problem than other editions of Lifemap. As one way to solve the AP handover problem, we think that contact duration and intercontact time between people and places could act as a complementary metrics because these two metrics are not influenced by the handover of APs. And these kind of metrics also make sense when it comes to routing protocols in MANET or VANET using landmarks as a relay such as [25] because they could provide information regarding contact between mobile nodes and those landmarks.

Figures 9 and 10 show the CCDF of contact duration and inter-contact time between people and places of our proposed model, CMM and different editions of Lifemap in terms of time period. In these figures, the results of all editions of Lifemap dataset are close which explains there is no influence from the AP handover problem. As shown

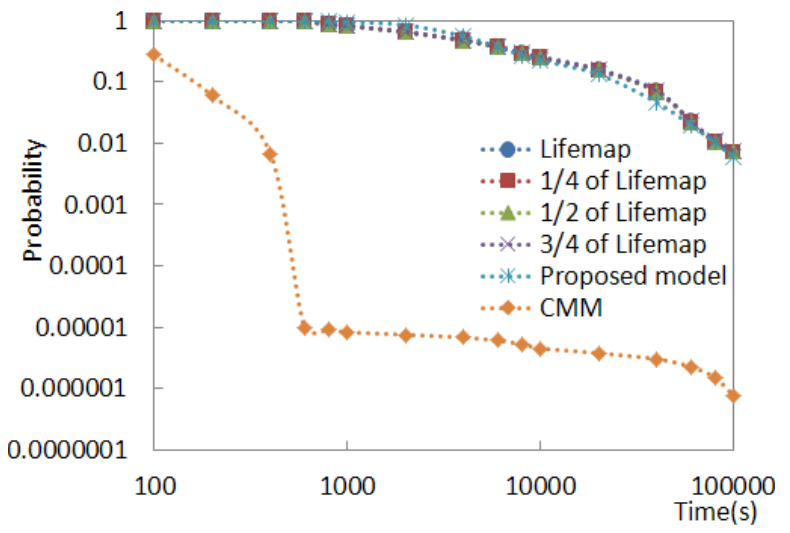

Fig. 9 Contact duration between people and places.

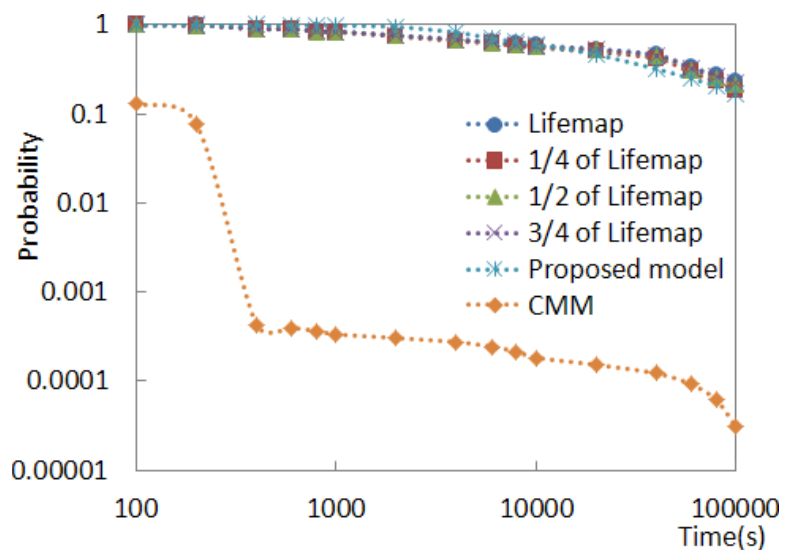

Fig. 10 Inter-contact time between people and places.

in these figures, the results of the proposed model are close to the results of compared real mobility. Because of CMM's deficiency in spatial and temporal properties, from these figures, the result of CMM is clearly far from the ones of the real mobility datasets.

\section{Conclusion}

In this paper, a mobility model based on relational graph as well as some new evaluation metrics and new mobility features were proposed. Relational graph is a graph which depicts relation between different objects, including relation between people and people, and between people and places. The introduction of relational graph improves the flexibility of mobility model while facilitating the usage of new evaluation metrics of contact duration and inter-contact time between people and places. In order to prove the results of our proposed mobility model, real mobility dataset of Lifemap. It was shown that our proposed model produced results similar to the Lifemap data.

If we would like to use our model in performance evaluation of network systems, first we would use a real mobility dataset to evaluate a network system, and then we could use a simulated mobility data (re)produced by the proposed model to further evaluate the network system. Even if the 
available datasets of real mobility is limited, we can produce a lot of datasets with the same characteristics as the real mobility, which would be helpful to ensure the generality of performance results.

Our future work is further comparative evaluations with other mobility models like HCMM, GeSoMo, and SWIM. Another future work is to consider the optimality $\alpha$ and $\beta$ in Eq. (4).

\section{Acknowledgments}

This work was supported in part by the National Institute of Information and Communications Technology (NICT), Japan.

\section{References}

[1] R. Shinkuma, H. Kasasi, K. Yamaguchi, and O. Mayora, "Relational metric: A new metric for network service and in-network resource control," 2012 IEEE Consumer Communications and Networking Conference (CCNC), pp.352-353, 2012.

[2] C. Lochert, H. Hartein, J. Tian, H. Fussler, D. Hermann, and M. Mauve, "A routing strategy for vehicular ad hoc networks in city environments," Proc. IEEE Intelligent Vehicles Symposium, pp.156$161,2003$.

[3] L. Pelusi, A. Passarela, and M. Conti, "Opportunistic networking: data forwarding in disconnected mobile ad hoc networks," IEEE Commun. Mag., vol.44, no.11, pp.134-141, 2006.

[4] M. Musolesi and C. Mascolo, "Designing mobility models based on social network theory," ACM SIGMOBILE Mobile Computing and Communications Review, vol.11, no.3, pp.59-70, 2007.

[5] D.B. Johnson and D.A. Maltz, "Dynamic source routing in ad hoc wireless networks," Kluwer International Series in Engineering and Computer Science, pp.153-179, 1996.

[6] V. Borrel, F. Legendre, M.D. Amorim, and S. Fdida, "Simps: Using sociology for personal mobility," IEEE/ACM Trans. Netw., vol.17, no.3, pp.831-842, 2009.

[7] Z. Ding, R. Shinkuma, and T. Takahashi, "Designing temporally and spatially integrated social mobility models for wireless network researches," 2013 15th Asia-Pacific. IEEE Network Operations and Management Symposium (APNOMS), pp.1-5, 2013.

[8] T. Hossmann, T. Spyropoulos, and F. Legendre, "Putting contacts into context: Mobility modeling beyond inter-contact times," Proc. Twelfth ACM International Symposium on Mobile Ad Hoc Networking and Computing, no.18, 2011.

[9] E. Cho, S.A. Myers, and J. Leskovec, "Friendship and mobility: user movement in location-based social networks," Proc. 17th ACM SIGKDD International Conference on Knowledge Discovery and Data Mining, pp.1082-1090, 2011.

[10] C. Boldrini, M. Conti, and A. Passarella, "Users mobility models for opportunistic networks: the role of physical locations," Proc. IEEE WRECOM, 2007.

[11] N. Vastardis and K. Yang, "An enhanced community-based mobility model for distributed mobile social networks," J. Ambient Intelligence and Humanized Computing, pp.1-11, 2012.

[12] D. Fischer, K. Herrmann, and K. Rothermel, "GeSoMo-general social mobility model for delay tolerant networks," 2010 IEEE 7th International Conference on Mobile Adhoc and Sensor Systems (MASS), pp.99-108, 2010.

[13] A. Gainaru, C. Dobre, and V. Cristea, "A realistic mobility model based on social networks for the simulation of VANETs," IEEE 69th Vehicular Technology Conference, 2009, VTC Spring 2009, pp.1-5, 2009.

[14] F. Ekman, A. Keranen, J. Karvo, and J. Ott, "Working day movement model," Proc. 1st ACM SIGMOBILE workshop on Mobility models, pp.33-40, 2008.

[15] W. Hsu, T. Spyropoulos, K. Psounis, and A. Helmy, "Modeling spatial and temporal dependencies of user mobility in wireless mobile networks," IEEE/ACM Trans. Netw., vol.17, no.5, pp.1564-1577, 2009.

[16] P. Venkateswaran, R. Ghosh, A. Das, S.K. Sanyal, and R. Nandi, "An obstacle based realistic ad-hoc mobility model for social networks," J. Networks, vol.1, no.2, pp.37-44, 2006.

[17] K. Lee, S. Hong, S.J. Kim, I. Rhee, and S. Chong, "Slaw: A new mobility model for human walks," IEEE INFOCOM 2009, pp.855$863,2009$.

[18] A. Mei and J. Stefa, "SWIM: A simple model to generate small mobile worlds," IEEE INFOCOM 2009, pp.2106-2113, 2009.

[19] J. Scott, Social Networks Analysis: A Handbook, Second ed. Sage Publications, London, United Kingdom, 2000.

[20] Y. Chon and H. Cha, "Lifemap: A smartphone-based context provider for location-based services," IEEE Pervasive Computing, vol.10, no.2, pp.58-67, 2011.

[21] U.S. Department of Transportation, Federal Highway Administration, 2009 National Household Travel Survey, URL: http://nhts.ornl.gov

[22] A.G. Ribeiro, R. Sofia, and A. Zuquete, "Improving mobile networks based on social mobility modeling," 19th IEEE International Conference on Network Protocols (ICNP), 2011, pp.289-291, 2011.

[23] D. Karamshuk, C. Boldrini, M. Conti, and A. Passarella, "Human mobility models for opportunistic networks," IEEE Commun. Mag., vol.49, no.12, pp.157-165, 2011.

[24] M. Musolesi, S. Hailes, and C. Mascolo, "Adaptive routing for intermittently connected mobile ad hoc networks," Sixth IEEE International Symposium on World of wireless mobile and multimedia networks, 2005, WoWMoM 2005, pp.352-353, 2005.

[25] S.M. Ahmed, M.E. Ali, and T. Hashman, Mobility Aware Data Delivery in Vehicular ad hoc Networks, Engine, 2013.

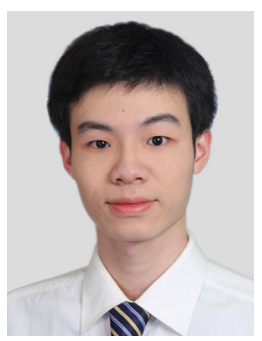

Zhenwei Ding received the B.E. degree in Information Engineering from Shanghai Jiao Tong University, Shanghai, China, in 2010. He is a master course student of Communications and Computer Engineering, Graduate School of Informatics, Kyoto University. His current research interest is in mobility models for wireless communication.

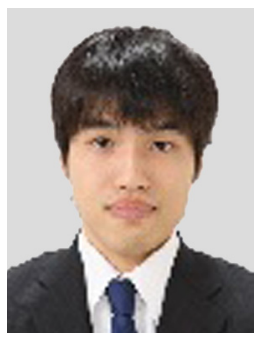

Yusuke Omori received the B.E. degree in Electrical and Electronic Engineering from Kyoto University, Kyoto, Japan, in 2013. He is a master course student of Communications and Computer Engineering, Graduate School of Informatics, Kyoto University. His current reseach is in analysis of social network. 


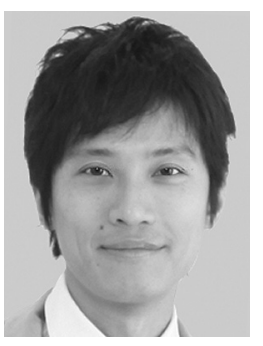

Ryoichi Shinkuma received the B.E., M.E., and Ph.D. degrees in Communications Engineering from Osaka University, Japan, in 2000, 2001, and 2003, respectively. In 2003, he joined the faculty of Communications and Computer Engineering, Graduate School of Informatics, Kyoto University, Japan, where he is currently an Associate Professor. He was a Visiting Scholar at Wireless Information Network Laboratory (WINLAB), Rutgers, the State University of New Jersey, USA, from 2008 Fall to 2009 Fall. His research interests include network design and control criteria, particularly inspired by economic and social aspects. He received the Young Researchers' Award from IEICE in 2006 and the Young Scientist Award from Ericsson Japan in 2007, respectively. He is a member of IEEE.

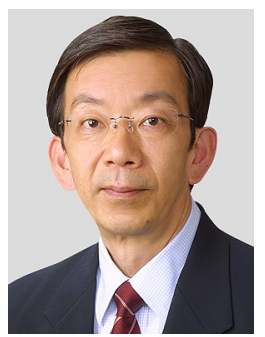

Tatsuro Takahashi received the B.E. and M.E. in Electrical Engineering from Kyoto University, Kyoto, Japan, in 1973 and 1975 respectively, and Dr. of Engineering in Information Science from Kyoto University in 1997. He was with NTT Laboratories from 1975 to 2000, making R\&D on high speed networks and switching systems for circuit switching, packet switching, frame relaying, and ATM. Since July 1, 2000, he is a Professor, Communications and Computer Engineering, Graduate School of Informatics, Kyoto University. His current research interests include high-speed networking, photonic networks and mobile networks. Prof. Takahashi received the Achievement Award from IEICE in 1996, the Minister of Science and Technology Award in 1998, and the Distinguished Achievement of Contributions Award from IEICE in 2011. He was a Vice President of the ATM Forum from 1996 to 1997, and the Chairman of the Network Systems (NS) Technical Group in the Communications Society of IEICE from 2001 to 2002. Prof. Takahashi is an IEEE Fellow. 